\title{
The Influence of Abnormal Mother to Infant Thyroid Function, Early Intervention Treatment on the Compensation for Mental Retardation
}

\author{
Zhao Ying ${ }^{1}$, Liu Xiaoqing ${ }^{1}$, Su Yang ${ }^{1}$, Zheng Ying ${ }^{1}$, Niu Guizhen ${ }^{2}$ \\ ${ }^{1}$ Department of Endocrinology, Jilin Central Hospital, Jilin, China \\ ${ }^{2}$ Jilin NCMS Management Center, Jilin, China
}

Email address:

zhaoying19630512@163.com (Zhan Ying)

\section{To cite this article:}

Zhao Ying, Liu Xiaoqing, Su Yang, Zheng Ying, Niu Guizhen. The Influence of Abnormal Mother to Infant Thyroid Function, Early Intervention Treatment on the Compensation for Mental Retardation. Clinical Medicine Research. Vol. 7, No. 4, 2018 , pp. 92-96. doi: $10.11648 /$ j.cmr.20180704.12

Received: July 9, 2018; Accepted: August 9, 2018; Published: October 11, 2018

\begin{abstract}
Objective: To observe the hyperthyroidism and hypothyroidism patients with pregnancy after production, the baby's thyroid function changes, the baby's mental abnormality, compensation for the children with intellectual disabilities and early intervention after treatment. Methods: In this study, 151 pregnant women with abnormal thyroid function from Department of Endocrinology in Jilin Central Hospital between 2010-2016, these women in the G12 and G24 were measured FT3, FT4, TSH, TPO, TG-ab, the babies at birth, 6 months, 12 months, examine their thyroid function, exercise and intelligence score respectively. Results: Hyperthyroidism group and hypothyroidism group of pregnant women after intervention treatment of G12 and G24, thyroid function tends to normal, there were no significant differences in FT3, FT4 and TSH levels. TSH was significantly increased in the patients with hyperthyroidism group at birth, compared with the other two groups, $\mathrm{P}<0.05$, the difference was statistically significant. Comparison of the nerve intelligence development and motor scores of the pregnant women in each group MDI and PDI, the levels of MDI and PDI in the hyperthyroidism group were decreased, but the late rise was obvious, there was no significant difference between the groups. There was no significant difference in the development of thyroid and nerve intelligence and motor scores after early intervention in the healthy control group. Conclusion: The abnormal thyroid function of pregnant women can affect the thyroid function of offspring, so that the intelligence development of offspring is affected. Adverse pregnancy was significantly increased, timely intervention treatment of pregnant women with abnormal thyroid function and adjustment of sub generation, the intelligence score and the absolute value of the motor score can be increased, it shows compensative effect on mental retardation in their offspring.
\end{abstract}

Keywords: Thyroid Function, Mental Retardation, Intervention Therapy

\section{Introduction}

The abnormality of thyroidal function during pregnancy can affect fetal growth and development and intellectual level $[1,2]$. The positive for thyroid peroxidase (TPO) or thyroglobulin (Tg) antibody [3, 4], and overt hyperthyroidism and overt hypothyroidism during pregnancy may impair the thyroid function and influence the growth and development of the offspring. It has been drawn much attention of researchers all over the world [5]. The objective of this study was to observe the hyperthyroidism and hypothyroidism patients with pregnancy after production, the baby's thyroid function changes, the baby's mental abnormality, compensation for the children with intellectual disabilities and early intervention after treatment, provide reference for clinical take effective care.

\section{Objectives and Methods}

\subsection{Objectives}

151 pregnant women from Department of Endocrinology 
in Jilin Central Hospital between 2010 and 2016 were included in the study. Our study included 50 pregnant patients with hyperthyroidism, 51 pregnant patients with hypothyroidism and 50 healthy pregnant women. All the patients were diagnosed according to clinical guidelines on the diagnosis and treatment of thyroid disease during pregnancy and the postpartum in China. If the patient is first diagnosed, reconfirm at another time. Eligible subjects consisted of pregnant women, who were free of clinical thyroid disorders, were not taking any thyroid medication (such as amiodarone), were permanent residents and to complete pregnancy and childbirth in our city, Subjects were also excluded if they had uncertained thyroid nodules or have connective tissue diseases and other autoimmune diseases. Meanwhile, 50 healthy pregnant women with thyroid autoantibody negative at between 12 and 30 weeks were as the control group.

\subsection{Methods}

\subsubsection{Groups}

151 pregnant women were divided into 3 groups. First group consisted of 50 patients with hyperthyroidism, 27 of whom had hyperthyroidism, 23 of whom had subclinical hyperthyroidism. Second group consisted of 51 patients with hypothyroidism, 22 of whom had hypothyroidism, 29 of whom had subclinical hypothyroidism. Control group included 50 subjects who had no previous history of thyroid disease. Patients with thyroid diseases were given corresponding treatment. 151 pregnant women were screened for thyroid function at G12 and G24. The peripheral blood from calcar pedis of the newborn was born within a week were tested thyroid-stimulating hormone(TSH). Thyroid function and mental developments were evaluated at 6th and 12th after birth. We applied Bayley Scales of Infant Development (BSID) to measure the mental development index (MDI) and psychomotor development index (PDI) of the children at age 6 months and 12 months. The work were completed by two testers, who have been trained to provide intelligence assessment. In order to avoid subjective deviation and ensure the quality of testing, our clinical study using single-blinded and taking the average scores of the two doctors' test results.

\subsubsection{Endocrine and Biochemical Indexes}

All pregnant women had thyroid function tests (serum TSH, FT3 and FT4) and thyroid autoimmunity tests (serum TgAb and TPOAb) at G12 and G24. The peripheral blood from calcar pedis of the newborn was born within a week were tested thyroid-stimulating hormone(TSH). Thyroid function and mental developments were evaluated at 6 th and 12 th after birth.

\subsubsection{Biochemical Assays}

Thyroid function tests (serum TSH, FT3 and FT4) and thyroid autoimmunity tests (serum TgAb and TPOAb) all performed in the same laboratory with the same corresponding kits by Abbott Laboratories, which are based on electroluminescence.

\subsection{Statistical Treatment}

The normal distribution data is represented by $\mathrm{x} \pm \mathrm{s}$ and the partial distribution data is represented by median. Paired sample $t$ test was used to compare the same index level at different time points. $\mathrm{P}<0.05$ was considered as a statistically significant difference.

\section{Results}

There were 50 cases of hyperthyroidism group, including 29 cases of hyperthyroidism, which included 1 case of hyperthyroidism crisis of pregnant women accounting for $3.45 \%(1 / 29), 2$ cases of premature birth accounting for $6.89 \%(2 / 29), 5$ cases of pregnancy-induced hypertension accounting for $17.24 \%(5 / 29)$ and 2 cases of termination of pregnancy accounting for $6.89 \%(2 / 29) .21$ cases of subclinical hyperthyroidism included 1 case of pregnancy-induced hypertension accounting for $4.76 \%$ $(1 / 21)$. In the offspring of pregnant women with hyperthyroidism, there was 1 case of SGA, 1 case of hypothyroidism, 1 case of subclinical hypothyroidism, 1 case of FT3 and FT4 elevation, and 2 cases of abnormal TPO or TG-ab, 1 case of growth hormone deficiency, 1 case of low birth weight infants, and 1 case of short stature. In the hyperthyroid group, the number of adverse pregnancies increased. The compliance of pregnant women was poor leading to the poor control of the thyroid function. And there was an increase of abnormal thyroid function, premature birth, SGA and short stature in offspring. The infants after early intervention had a significant increase in the development of thyroid function and neurological intelligence and exercise scores, but there was no significant difference between the control group and healthy control group. There were 51 cases of hypothyroid group, including 22 cases of hypothyroidism, 29 cases of subclinical hypothyroidism. And in their offspring, there were 8 cases of infants with abnormal thyroid function, 2 cases of abnormal TPO or TG-ab, and 1 infant whose height exceeded +2 SD, 2 infants exceeded +1 SD. There were 50 cases of healthy controls without abnormal thyroid function in their offspring, but including 2 cases abnormal TPO or $\mathrm{TG}-\mathrm{ab}$ in their offspring.

\subsection{Comparison of Thyroid Function of G12 and G24 in Pregnant Women in Various Groups}

After the intervention treatment of pregnant women, the thyroid function in the hyperthyroidism and hypothyroidism groups tended to be normal. There was no statistical difference of the levels of FT3, FT4, and TSH in each group between G12 and G24 ( $\mathrm{P}>0.05)$, indicating that intervention therapy was effective and avoided the impact of disease on offspring. Showed in Table 1. 
Table 1. Comparison of thyroid function in pregnant women invarious groups ( $\bar{X} \pm s)$.

\begin{tabular}{llllll}
\hline Group & Number & FT3 pmol/l & FT4 pmol/l & TSH mIU/L \\
\hline \multirow{2}{*}{ Hyperthyroidism group } & \multirow{2}{*}{50} & G12 & $3.211+-1.22$ & $14.551+-2.68$ & $1.716+-1.34$ \\
& & G24 & $3.542+-1.41$ & $13.041+-2.41$ & $2.152+-1.29$ \\
Hypothyroidism group & \multirow{2}{*}{51} & G12 & $3.635+-1.29$ & $14.064+-2.65$ & $2.645+-1.05$ \\
\multirow{2}{*}{ Control group } & & G24 & $3.712+-1.07$ & $12.564+-3.28$ & $2.742+-0.91$ \\
& \multirow{2}{*}{50} & G12 & $4.148+-0.85$ & $13.865+-3.03$ & $1.476+-0.99$ \\
& & G24 & $4.170+-1.31$ & $12.712+-2.51$ & $1.85+-0.92$ \\
\hline
\end{tabular}

G12: Pregnant for 12 weeks, G24: Pregnant for 24 weeks

\subsection{Comparison of Thyroid Function of Infants in Each Groups}

The TSH level of infants born by hyperthyroidism group at birth was significantly increased compared with other two groups $(\mathrm{P}<0.05)$. There was no significant difference between
A6 and A12. There was no significant difference of FT3 and FT4 levels between three groups $(\mathrm{P}>0.05)$, indicating that the intervention treatment of pregnant women was beneficial to the offspring's thyroid function. Showed in Table 2.

Table 2. Comparison of newborn thyroid function at different stages after birth invarious groups ( $\bar{X} \pm s)$.

\begin{tabular}{llllll}
\hline Group & Number & FT3 pmol/l & & FT4 pmol/l & TSH $\mathbf{~ I U / L ~}$ \\
\hline \multirow{3}{*}{ Hyperthyroidism group } & \multirow{3}{*}{50} & A0 & $3.232+-1.89$ & $11.745+-2.10$ & $8.113+-2.63^{*}$ \\
& & A6 & $3.621+-1.32$ & $13.121+-2.4$ & $3.414+-1.09$ \\
& & A12 & $3.951+-1.21$ & $14.041+-2.51$ & $2.152+-0.93$ \\
Hypothyroidism group & \multirow{2}{*}{51} & A0 & $3.975+-1.40$ & $14.562+-2.43$ & $1.468+-0.91$ \\
& & A6 & $3.435+-1.35$ & $13.167+-2.17$ & $1.836+-1.45$ \\
Control group & & A12 & $3.882+-1.23$ & $13.835+-2.13$ & $1.53+-1.32$ \\
& \multirow{2}{*}{50} & A0 & & & $1.643+-0.89$ \\
& & A6 & $4.089+-1.21$ & $12.990+-2.10$ & $3.01+-1.05$ \\
\end{tabular}

A0: born A6: born 6 months A12: born 12 months

$*: \mathrm{p}<0.05$

\subsection{Comparison the Neurological Intelligence (MDI) and Motor Scores(PDI) of Offspring in Each Group of Pregnant Women}

The levels of MDI and DPI in the hyperthyroidism group decreased compared with other two groups at A6, but increased significantly at A12 compared with A6. There were differences among the groups, but there was no statistical significance. Showed in Table 3 and Table 4.

Table 3. Comparison of MDI of the children of the pregnant women in various groups ( $\bar{X} \pm s)$.

\begin{tabular}{llllll}
\hline Group & Number & $\overline{\mathbf{X}} \pm \mathbf{s}$ & & Median range & Interquartile \\
\hline \multirow{2}{*}{ Hyperthyroidism group } & \multirow{2}{*}{50} & A6 & $115.24 \pm 11.06$ & 115 & $113-126$ \\
& & A12 & $117.56 \pm 16.07$ & 119 & $104-135$ \\
Hypothyroidism group & \multirow{2}{*}{51} & A6 & $119.67 \pm 11.63$ & 120 & $112-126$ \\
& & A12 & $117.01 \pm 10.32$ & 119 & $111-126$ \\
Control group & \multirow{2}{*}{50} & A6 & $116.56 \pm 10.53$ & 118 & $113-135$ \\
& & A12 & $118.78 \pm 12.01$ & 119 & $113-127$ \\
\hline
\end{tabular}

MDI: mental devdlopment index

Table 4. Comparison of PDI of the children of the pregnant women in various groups $(\bar{X} \pm s)$.

\begin{tabular}{llllll}
\hline Group & Number & $\overline{\mathbf{X}} \pm \mathbf{s}$ & & Median range & Interquartile \\
\hline \multirow{2}{*}{ Hyperthyroidism group } & \multirow{2}{*}{50} & A6 & $115.24 \pm 11.02$ & 115 & $113--126$ \\
& & A12 & $118.56 \pm 16.07$ & 119 & $104-135$ \\
Hypothyroidism group & \multirow{2}{*}{51} & A6 & $117.67 \pm 10.17$ & 120 & $112-126$ \\
& & A12 & $119.01 \pm 11.32$ & 119 & $111-126$ \\
Control group & \multirow{2}{*}{50} & A6 & $116.56 \pm 12.53$ & 118 & $103-135$ \\
& A12 & $118.78 \pm 15.01$ & 119 & $113-127$ \\
\hline
\end{tabular}

PDI: psychomoter devdlopment index 


\section{Discuss}

\subsection{Abnormal Thyroid Function During Pregnancy Has an Effect on the Fetal and Postnatal Thyroid Function}

Hyperthyroidism, hypothyroidism, antibody positive and low T4 concentration had a great influence on newborn, pregnant women at antenatal examination found that TSH in patients with abnormal timely medical treatment, observe its change, avoid fluctuations, after birth, we also can reduce adverse pregnancy outcomes, and children's abnormal development of the nervous system and mental development lag behind, active treatment of infant intelligence obstacle has compensation function.

\subsection{To Recognize the Importance of Abnormalities in Pregnancy}

Improve the understanding of pregnancy with abnormal thyroid function, for proper diagnosis and treatment, will significantly improve the prognosis of the mother and the fetus to ensure the health of women, each of thyroid disease during pregnancy women, we must regularly detection, abnormal treatment, postpartum follow-up is very important.

\subsection{Pregnant Women with Hyperthyroidism Have a Greater Impact on the Fetus}

Hyperthyroidism during pregnancy, a larger impact on the fetus, which can cause neonatal hyperthyroidism, intrauterine retardation, prolonged labor and SGA [6-7], Millar[8] the study found that the incidence of hyperthyroidism during pregnancy SGA is 9 times that of normal pregnancy, maternal TRAb is high enough, can cause neonatal hyperthyroidism, mainly TSAb can cause fetal hypothyroidism.

\subsection{Evaluating Thyroid Function Correctly in Early Trimester of Pregnancy Is Beneficial to the Growth and Development of the Fetus After Birth}

Maternal thyroid hormone levels to ensure the mother during pregnancy, and their children's health is very important, Abnormal maternal thyroidism can bring adverse outcomes to maternal pregnancy and fetal development, such as gestational hypertension, premature birth, abortion, low birth weight, offspring neural developmental damage, etc. Haddow etc and POP research results show that the first half of pregnancy maternal hypothyroidism. (Subclinical hypothyroidism. low T4) can also affect offspring nerve mental development, bring down the generations intelligence score. Therefore, during pregnancy, especially in the early weeks of pregnancy timely and correctly evaluate the status of thyroid function for pregnant women, is very important to the maternal and fetal health.

\subsection{Early Intervention on Pregnant Mothers Can Affect Pregnancy Outcomes and Fetal Thyroid Function}

Intervention during pregnancy, mother and baby thyroid function. Abnormal thyroid function including the mother during pregnancy low thyroid hormone levels, antibody positive or higher TSH levels, or only specific antibodies, thyroid, etc., will affect the development of the baby's nervous spirit, at the same time increase the miscarriage, premature birth and low birthweight nerve mental development, at the same time, increased risk of miscarriage, premature birth and low birth weight [9]. So some scholars suggested for TPO positive' women, a daily supplement L T450ug during the pregnancy and to reduce the risk of pregnancy hypothyroidism happened [10]. Different view, in iodine sufficient areas, normal reference range of serum T4 levels and mother TPOAb, TSH, T4 levels without correlation [11], and neonatal thyroid function has nothing to do with the mother during pregnancy - T4 L replacement therapy [12]. The study found that human intervention to make its own antibody positive pregnancy thyroid function to maintain in certain range, the fetal umbilical cord blood TSH level is still higher than the control group at birth, but not obvious T3, T4 levels drop. The intervention group of pregnancy thyroid function is lower than the control group, the fetal umbilical cord blood TSH level at birth and at the same time, accompanied by FT3, FT4 level was significantly lower than control group, and neonatal serum TSH is still higher than the control group and intervention group, and into the TSH level of infancy is still visible to the higher than the control group. Thus different thyroid functional status of pregnant women, the offspring born after thyroid function. Intervention during pregnancy have different effects on fetal thyroid function after birth [13].

\section{Conclusion}

The pregnant woman with abnormal thyroid function can affect the thyroid function of offspring, made the intelligence development of children affected. Adverse pregnancy hyperthyroidism group was obviously increased, The pregnant woman obedience is bad, her thyroid function control is not good, Abnormal thyroid function of infants increased, premature birth, SGA, dwarfisms increased. Our early intervention after the baby we and nervous mental development and movement score increased significantly, and the health control group no significant difference; Hypothyroidism group of pregnant women studies have shown that pregnant woman with autoimmune thyroid disease in pregnancy and infant in months for one month when Fine motor score significantly lower than normal babies, at the same time in infants aged for three months, six months and 12 months respectively were evaluated about Should things cans, Should people cans, Fine motor cans, Coarse action cans. Compared with healthy infants, the difference is significant and statistically significant $(\boldsymbol{p}<0.05)$. But there was no significant difference in verbal ability between children and healthy infants. Baby after birth, Should things cans development and pregnant woman are suffering from disease, pregnant mother of thyroid function status during the pregnancy and pregnant women' gestational age, history of medicine during pregnancy, maternal and 
infant body after the baby was born anti-thyroid antibodies has a direct relationship. The baby should be one with the baby's thyroid function, motherhood disease as well as the history of medicine has a direct relationship; Baby's Coarse action cans with the baby's thyroid function, baby TSAb and pregnant woman. Thyroid function during pregnancy has a direct relationship between [2]; The fine movements of infants are directly related to the thyroid function of infants and the thyroid function of pregnant mothers. We found that pregnant women with abnormal thyroid function timely intervention treatment. Reduced adverse pregnancy outcomes, the abnormal pregnant women children's intelligence scores and sports score rise in absolute value, compensation for the offspring of mental retardation.

\section{References}

[1] Tiemeier H, Peeters RP. Association of maternal thyroid function during early pregnancy with offspring IQ and brain morphology in childhood: a population-based prospective cohort study. Lancet Diabetes Endocrinol. 2016 Jan;4(1):35-43. doi: 10.1016/S2213-8587(15)00327-7.

[2] Levie D, Korevaar TIM, Bath SC, Dalmau-Bueno A, Murcia M, Espada M, Dineva M, Ibarluzea JM, Sunyer J, Tiemeier H, Rebagliato M, Rayman MP, Peeters RP, Guxens M. Thyroid Function in Early Pregnancy, Child IQ, and Autistic Traits: a Meta-analysis of Individual-participant Data. J Clin Endocrinol Metab. 2018 May 10. doi: 10.1210/jc.2018-00224.

[3] Derakhshan A, Korevaar TIM, Taylor PN, Levie D, Guxens M, Jaddoe VWV, Nelson SM, Tiemeier H, Peeters RP. The Association of Maternal Thyroid Autoimmunity During Pregnancy with Child IQ. J Clin Endocrinol Metab. 2018 Jul 17 doi: $10.1210 /$ jc.2018-00743.
[4] De Leo S, Pearce EN. Autoimmune thyroid disease during pregnancy. Lancet Diabetes Endocrinol. 2018 Jul;6(7):575-586. doi: 10.1016/S2213-8587(17)30402-3.

[5] Korevaar TIM, Tiemeier H, Peeters RP. Clinical associations of maternal thyroid function with foetal brain development: Epidemiological interpretation and overview of available evidence. Clin Endocrinol (Oxf). 2018 Apr 24. doi: 10.1111/cen.13724.

[6] Mannisto T. Mendola P. Grewal J. et al. Thyroid diseases and adverse pregnancy outcomes in a contemporary US cohort [J]. J Clin Endcrinol Metab, 2013, 98(7: 2725-2733).

[7] Andersen SL, Olsen J, Wu CS, et al. Spontaneous abortion, stllbirth and hyperthyroidism: a danish population-based study [J]. Eur Thyroid J, 2014, 3(3):164-172.

[8] Millar LK, Wing DA, Leung AS, et al, Low birth weight and preeclampsia in pregnancies complicated by hyperthyroidism [J]. Obstet Gynecol, 1994, 84(6):946-949.

[9] Gartner R. Thyroid diseases in pregnancy. Lurr Opin Obstet Gynecol, 2009, 67:178-182.

[10] Debieve F, Duliere S, Bemard P, et, al. To treat or not to treat euthyroid autoimmmmune disorder during pregnancy? Gynecol Obestet Invest, 2009, 67:178-182.

[11] Oken E, BRAVERMAN 1E, Plate D, et al. Neonatal Thyroxine, maternal thyroid function, and chijd cognition. J Clin Endocrinol Metab, 2009, 94:497-503.

[12] Rovelli R, Vigone MC, Giovanettoni C, et al. Newborn of mothers affected autoimmune thyroiditis: the importance of thyroid function monitoring in the first months of life. Ital J Pediatr, 2010, 36: 24.

[13] Du Peiyi, kitta Zhong Lili, etc. Thyroid autoantibody positive women intervention effect on infants thyroid function during pregnancy. The endocrine metabolism, 2010, 26:931-935. 